\title{
Manejo de diabetes mellitus tipo 2 con análogos GLP-1: una experiencia real
}

\author{
Eduardo José Polanía1, Guillermo E. Guzmán², Veline Martínez Calvache³, Karen Fériz², \\ Carolina García ${ }^{1}$, Alisson Tabares ${ }^{4}$, Natalia Pardo ${ }^{4}$
}

${ }^{1}$ Universidad ICESI, Facultad de Medicina, residente
medicina interna.
${ }^{2}$ Universidad ICESI, docente de endocrinología. Departa
Endocrinología, Fundación Valle del Lili, Cali, Colom
${ }^{3}$ Universidad ICESI, docente de medicina interna. Depar
de Medicina Interna, Fundación Valle del Lili, Cali, C
${ }^{4}$ Universidad ICESI, Facultad de Medicina, estudiantes
medicina.
Correspondencia
Veline Martínez
Departamento de Medicina Interna
Fundación Valle del Lili,
Cali, Colombia
Correo electrónico: velinemartinez@gmail.com
Móvil:+57 3006189469

\section{Responsabilidades éticas}

Protección de personas y animales. Los autores declaran que para esta investigación no se han realizado experimentos en seres humanos ni en animales.

Confidencialidad de los datos. Los autores declaran que han seguido los protocolos de su centro de trabajo sobre la publicación de datos de pacientes.

Derecho a la privacidad y consentimiento informado. Los autores declaran que en este artículo no aparecen datos de pacientes.

Financiación: Ninguna.

Conflicto de intereses: Los autores declaran no tener ningún conflicto de intereses.

Fecha de recepción: 11/12/2017

Fecha de aceptación: 26/01/2018

\section{Resumen}

Introducción: La diabetes mellitus tipo 2 (DM2) es un problema sanitario importante en la actualidad, con alta morbilidad, mortalidad, costos directos e indirectos. Nuevas estrategias de manejo se han implementado para disminuir su impacto. Este estudio describe la experiencia del manejo con análogos GLP-1.

Métodos: Estudio retrospectivo, descriptivo, de pacientes con diagnóstico de DM2 en manejo con análogos GLP-1. Se describieron características clínicas al inicio de la terapia y control entre 3 -y6 meses.

Resultados: De 69 pacientes, 55,1\% eran mujeres, con edad promedio 57,1 años. La mediana de duración de DM2 fue 13 años. Se encontró reducción significativa del peso $3 \mathrm{~kg}$ $(\mathrm{p}=0,00), \operatorname{HbA} 1 \mathrm{c} 1,7 \%(\mathrm{p}=0)$, glucemia en ayunas $41 \mathrm{mg} / \mathrm{dL}$ $(\mathrm{p}=0)$, colesterol total $14 \mathrm{mg} / \mathrm{dL}(\mathrm{p}=0,0069)$ y triglicéridos 38 $\mathrm{mg} / \mathrm{dL}(\mathrm{p}=0,0247)$. No se presentaron cambios significativos en la tensión arterial, colesterol HDL y LDL.

Conclusión: Los análogos GLP-1 en pacientes con DM2 generan mejoría significativa en control glucémico, peso y colesterol. Es necesario un seguimiento a largo plazo para determinar mantenimiento del control glucémico, pérdida de peso e impacto en el riesgo cardiovascular.

Palabras clave: análogos de GLP-1, diabetes mellitus tipo 2, incretinas.

\section{Summary}

Introduction: Type 2 Diabetes Mellitus (DM2) is an important health problem at present, with high morbidity, mortality, direct and indirect costs. New management strategies have been implemented to reduce their impact. This study describes the experience of handling GLP-1 analogs.

Methods: Retrospective, descriptive study of patients with DM2 on management with GLP-1 analogues. Clinical characteristics were described at the beginning of therapy and control between 3 and 6 months.

Results: Of 69 patients, $55.1 \%$ were women, the average age was 57.1 years. The median duration of DM2 was 13 years. A significant reduction of weight $\mathrm{kg}(p=0.00)$, the $\mathrm{HbA1c} 1.7 \%$ $(p=0)$, the fasting glycemia $41 \mathrm{mg} / \mathrm{dL}(p=0)$, the total cholesterol $14 \mathrm{mg} / \mathrm{dL}(P=0.0069)$, the triglycerides $38 \mathrm{mg} / \mathrm{dL}$ (0.0247) was found. There were no significant changes in blood pressure, HDL and LDL cholesterol.

Conclusion: GLP-1 analogs in patients with T2DM generate significant improvement in glycemic control, weight and cholesterol. Long-term follow-up is necessary to determine maintenance of glycemic control, weight loss and impact on cardiovascular risk.

Keywords: GLP-1 analogs, type 2 diabetes mellitus, incretins. 


\section{Introducción}

La diabetes mellitus tipo 2 (DM2) es uno de los problemas sanitarios más importantes en la actualidad. Su prevalencia ha aumentado en forma exponencial en el mundo entero, con alta morbilidad, mortalidad, costos directos e indirectos, por lo que un buen control de la patología es fundamental ${ }^{(1)}$. La enfermedad tiene un carácter progresivo, por lo que es necesaria la intensificación del tratamiento en la mayoría de los pacientes para lograr las metas en el control y, por ende, reducción de las complicaciones ${ }^{(2,3,4)}$. Los análogos GLP-1 constituyen una herramienta terapéutica novedosa disponible en nuestro medio para este fin ${ }^{(5,6)}$. Los estudios han mostrado que este grupo de medicamentos pueden llevar a mayor reducción de la HbA1c (hemoglobina glucosilada), asociado a pérdida de peso, mejoría en el perfil lipídico, además de un efecto cardioprotector contra el daño isquémico y la insuficiencia cardia$\mathrm{ca}^{(7,8,9)}$. A pesar de ser una estrategia importante en el manejo actual de la DM2, cuya eficacia se ha visto reflejada en estudios a nivel mundial, en Colombia no hay estudios que evalúen la respuesta de estos medicamentos en nuestra población. Este estudio tuvo por objetivo describir la experiencia inicial del uso de análogos GLP-1 en el manejo de pacientes con DM2 en la Fundación Valle del Lili, entre 2013 y 2016, medida en términos de HbA1c, peso y perfil lipídico.

\section{Métodos}

Se realizó un estudio descriptivo y retrospectivo, de pacientes adultos de ambos sexos, con diagnóstico de DM2, definida de acuerdo con los criterios propuestos por la guía de la Asociación Americana de Diabetes 2016 $6^{(10)}$, atendidos en consulta externa de endocrinología de la Fundación Clínica Valle del Lili, entre 2013 y 2016, institución de alto nivel de complejidad. Fueron elegibles pacientes a quienes se les prescribió un análogo GLP-1 como parte del manejo de la enfermedad, durante un periodo no menor a tres meses y para lograr mayor uniformidad en los resultados que no fuera mayor a seis meses. Se excluyeron pacientes embarazadas. La población de estudio fue seleccionada a partir del registro de estadística de la institución de acuerdo con los códigos CIE 10 (E10 - E149) relacionados con diabetes mellitus y sus complicaciones. Se construyó una base de datos para registro electrónico en el programa Microsoftt ${ }^{\circledR}$ Excel $^{\circledR}$ 2016. Se incluyeron variables demográficas como edad, sexo, tipo de afiliación al sistema de salud, antecedentes médicos como hipertensión, dislipidemia, enfermedad coronaria. Así mismo, variables del examen físico, como peso, talla, nivel de tensión arterial; estudios de laboratorio, como nivel de HbA1c, glucemia en ayunas y perfil lipídico. Las variables del examen clínico y de laboratorio se midieron al inicio del manejo con análogos GLP-1 y en el control a los tres meses de iniciada la medicación.
Para el análisis estadístico, las variables cuantitativas fueron reportadas como promedio o mediana, y medidas de dispersión, desviación estándar y rango intercuartílico de acuerdo con el cumplimiento de supuestos de normalidad. Las variables categóricas se describieron como valor absoluto y porcentaje. Se compararon cada una de las variables, las cuantitativas con la prueba t de Student o Wilcoxon Mann Whitney y las categóricas con Chi cuadrado o test exacto de Fisher según el cumplimiento de supuestos. Los análisis estadísticos se hicieron en STATA $12^{\circledR}$. El presente estudio tuvo aprobación del comité de ética en investigación biomédica de la institución.

\section{Resultados}

Se obtuvieron 2.727 individuos con diagnóstico de DM2 en los registros hospitalarios, en el periodo de tiempo comprendido entre enero de 2013 y diciembre de 2016, de estos se seleccionaron 169 que tenían manejo con análogos GLP-1, de los cuales 69 continuaron seguimiento y contaban con los paraclínicos necesarios para el análisis (figura 1). Las características de la población se presentan en la tabla 1. La edad promedio fue 57,1 años y el 55,1\% eran mujeres. El 56,5\% de los pacientes pertenecían al régimen contributivo. Tenían antecedente de hipertensión arterial 71\%, dislipidemia 55,1\% y enfermedad coronaria $15,9 \%$ (tabla 1).

Figura 1. Flujograma de pacientes en el estudio con diagnóstico de diabetes según CIE-10: Clasificación Internacional de Enfermedades $1^{\circ}$, códigos: E10 - E149

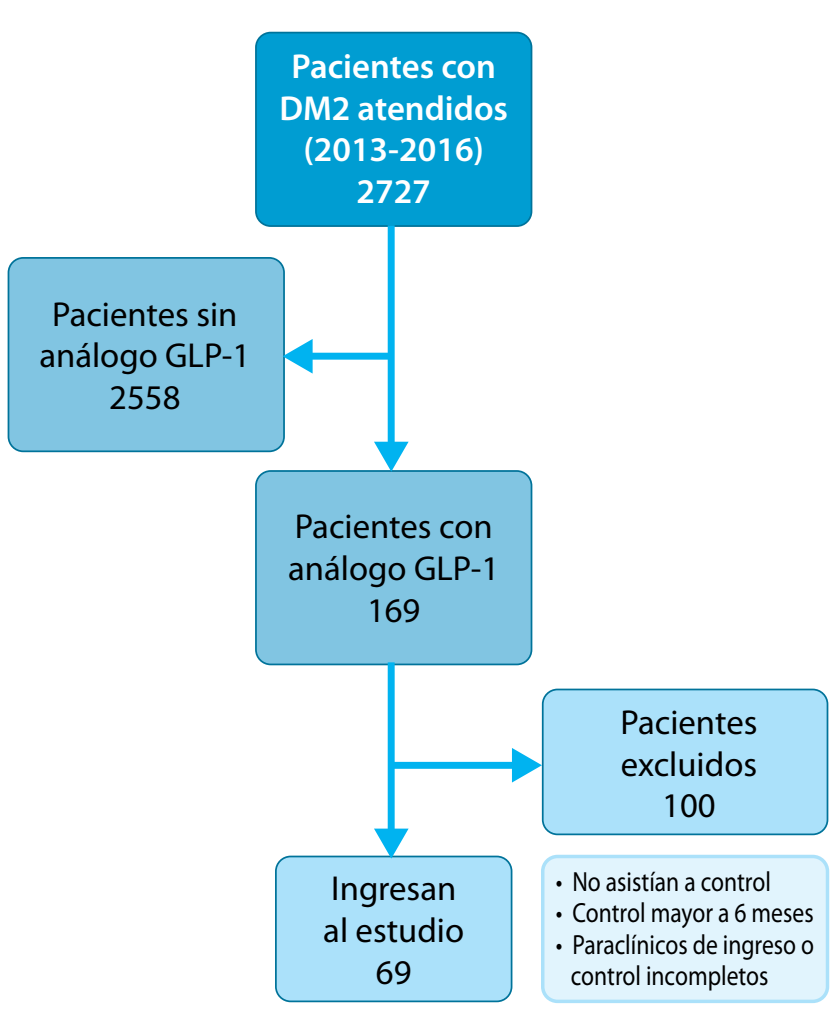


Tabla 1. Características generales y antecedentes de los pacientes

\begin{tabular}{|c|c|}
\hline Característica & $\begin{array}{c}\text { Total pacientes } \\
\mathrm{n}=69(\%)\end{array}$ \\
\hline Edad* & $57,1 \pm 10,5$ \\
\hline \multicolumn{2}{|l|}{ Sexo } \\
\hline Femenino & $38(55,1)$ \\
\hline Masculino & $31(44,9)$ \\
\hline \multicolumn{2}{|l|}{ Régimen de salud } \\
\hline Subsidiado & $1(1,5)$ \\
\hline Contributivo & $39(56,5)$ \\
\hline Prepagado & $28(40,6)$ \\
\hline Especial & $1(1,5)$ \\
\hline \multicolumn{2}{|l|}{ Comorbilidades } \\
\hline Hipertensión arterial & $49(71)$ \\
\hline Dislipidemia & $38(55,1)$ \\
\hline Enfermedad coronaria & $11(15,9)$ \\
\hline Tiempo con diagnóstico de DM2, meses** & $156(60-210)$ \\
\hline \multicolumn{2}{|l|}{ Tratamiento DM2 } \\
\hline Antidiabético oral & $15(21,74)$ \\
\hline Insulina & $50(72,46)$ \\
\hline Sin tratamiento previo & $4(5,8)$ \\
\hline \multicolumn{2}{|l|}{ Uso de estatinas } \\
\hline No & $18(26,1)$ \\
\hline Sí & $51(73,9)$ \\
\hline \multicolumn{2}{|l|}{ Análogo de GLP-1 } \\
\hline Exenatide & $1(1,5)$ \\
\hline Liraglutide & $25(36,2)$ \\
\hline Dulaglutide & $3(4,4)$ \\
\hline Exenatide QW & $40(58)$ \\
\hline \multicolumn{2}{|l|}{ Efectos adversos } \\
\hline Pancreatitis & $0(0)$ \\
\hline Vómito & $0(0)$ \\
\hline Náuseas & $5(7,3)$ \\
\hline Diarrea & $0(0)$ \\
\hline Abandono de tratamiento & $5(7,3)$ \\
\hline \multicolumn{2}{|l|}{ Características clínicas pre GLP-1 } \\
\hline Peso** & $85(72-97) \mathrm{kg}$ \\
\hline Talla* & $164,42 \pm 9 \mathrm{~cm}$ \\
\hline $\mathrm{IMC}^{*}$ & $37,47 \pm 4,46$ \\
\hline
\end{tabular}

Valores expresados como valor absoluto (porcentaje).* Reportado como media (desviación estándar). ${ }^{* *}$ Reportado como mediana (rango intercuartílico). DM2: diabetes mellitus tipo 2. Análogos GLP-1: análogos del péptido similar al glucagón tipo 1. IMC: índice de masa corporal.
La mediana de tiempo con diagnóstico de DM2 era 156 meses; el 74,47\% de los pacientes recibían manejo con insulina, $21,73 \%$ antidiabéticos orales y $5,8 \%$ de entrada fueron manejados con este tipo de medicamento. Fueron prescritos con exenatide QW (Bydureon) 58\%, liraglutide (Victoza) $36,2 \%$. Presentaron efectos adversos a la medicación 5 pacientes $(7,3 \%)$, el más frecuente fue náuseas (100\% de los casos), lo que llevó al abandono de la medicación luego de tres meses de manejo, razón por la que no fueron excluidos del análisis.

Se documentó reducción de 1,7\% en la HbA1c mientras que la glucemia en ayunas pasó de $153 \mathrm{mg} / \mathrm{dL}$ a $112 \mathrm{mg} / \mathrm{dL}$ (41 $\mathrm{mg} / \mathrm{dL}$ ), siendo estos hallazgos significativos $(\mathrm{p}<0,05)$. A los tres meses de inicio de análogos GLP-1, 46,3\% de los pacientes tenían HbA1c menor de 7. A ocho pacientes con HbA1c menor a $7 \%$, se inició la medicación, por la necesidad de intensificar metas según criterio del tratante, que continuaron controlados.

La reducción de peso y del índice de masa corporal fueron significativas, siendo estos efectos un poco mayores en hombres. En relación al perfil lipídico, se encontró reducción significativa en el colesterol total, de $13,5 \mathrm{mg} / \mathrm{dL}$ y de los triglicéridos $(38 \mathrm{mg} / \mathrm{dL}$ ), sin cambios significativos en colesterol LDL (c-LDL) ni colesterol HDL (c-HDL). La reducción de la presión arterial sistólica y diastólica no fue significativa (tabla 2).

\section{Discusión}

La DM2 corresponde al $90 \%$ de todos los pacientes con diabetes mellitus en el mundo y resulta ser una epidemia tanto en países desarrollados como en los subdesarrollados ${ }^{(11,12,13)}$; es una patología con impacto importante en la morbilidad y mortalidad por las complicaciones que se derivan de ella. Los análogos de GLP-1 son una estrategia de manejo novedosa para esta enfermedad, se ha demostrado que pueden llevar a una reducción de HbA1c entre 0,8-1,9\%, efecto que parece depender de la molécula usada ${ }^{(14)}$. Se calcula que, de los pacientes manejados en la institución para el momento del estudio, cerca del 7\% tenían como parte del tratamiento este tipo de medicamentos. En nuestro, caso la reducción global de HbA1c fue 1,7\% comparable con los resultados de ensayos clínicos. De los pacientes estudiados al inicio de manejo con análogo GLP-1, la mayoría tenía insulina como parte del tratamiento, el 65,22\% usaba esquema basal-bolo, grupo en el que se documentó una reducción significativa de la HbA1c y la glucemia en ayunas, similar a lo descrito por otros autores ${ }^{(15,16,17,18,19)}$ (tabla 3).

Se ha demostrado que exenatide semanal y liraglutide pueden reducir la HbA1c en 1,5\% ${ }^{(20,21)}$; en nuestro estudio, la disminución registrada fue de $2 \%$ y $1,3 \%$, respectivamente (tabla 4), diferencia que podría ser explicada por el valor mayor de HbA1c al inicio de los pacientes expuestos a exenatide vs. liraglutide, pues es ya conocido que entre más alto sea el nivel de HbA1c al inicio del tratamiento, mayor será el descenso de la misma ${ }^{(22)}$. Del análisis individual se excluyeron dulaglutide 
Tabla 2. Datos clínicos y paraclínicos comparativos pre y post- GLP-1

\begin{tabular}{|c|c|c|c|}
\hline $\begin{array}{c}\text { Variable } \\
n=69\end{array}$ & Pre- GLP-1 & Post- GLP-1 & $\mathbf{p}$ \\
\hline Peso $(\text { en } \mathbf{k g})^{* *}$ & $85(72-97)$ & $82(71-95)$ & 0,00 \\
\hline Mujeres** & $76(69-85)$ & $75,5(68-84)$ & 0,0001 \\
\hline Hombres** & $97(86-103)$ & $93(82-100)$ & 0,0006 \\
\hline IMC* & $37 \pm 4,46$ & $30,63 \pm 4,49$ & 0,00 \\
\hline Mujeres** & $31 \pm 4,12$ & $30,2 \pm 4,2$ & 0,0053 \\
\hline Hombres** & $32 \pm 4,8$ & $31,6 \pm 4,8$ & 0,0014 \\
\hline Tensión arterial sistólica $(\mathrm{mmHg})^{* *}$ & $120(110-130)$ & $120(110-130)$ & 0,9152 \\
\hline Mujeres** & $120(100-130)$ & $120(110-120)$ & 0,334 \\
\hline Hombres** & $120(110-130)$ & $120(110-130)$ & 0,3748 \\
\hline Tensión arterial diastólica $(\mathrm{mmHg}) * * * *$ & $79(65-80)$ & $80(70-80)$ & 0,6073 \\
\hline Mujeres** & $74(60-80)$ & $70(60-80)$ & 0,474 \\
\hline Hombres** & $80(70-80)$ & $80(70-80)$ & 0,1985 \\
\hline HbA1c\% ** & $8,7(7,7-9,9)$ & $7(6,5-7,85)$ & 0,00 \\
\hline Mujeres** & $9,4(8-9,9)$ & $7,1(6,7-7,8)$ & 0,00 \\
\hline Hombres** & $8,1(7,55-9,5)$ & $6,95(6,3-8,1)$ & 0,0002 \\
\hline Glucemia en ayunas $(\mathrm{mg} / \mathrm{dL}) * *$ & $153(117-202)$ & $112(97-147)$ & 0,00 \\
\hline Mujeres** & $167,5(122-201)$ & $109(103-146)$ & 0,001 \\
\hline Hombres** & $145(117-203)$ & $113(87-158)$ & 0,0031 \\
\hline Colesterol total $(\mathrm{mg} / \mathrm{dL}) * *$ & $162(139-199)$ & $148,5(132-184)$ & 0,0069 \\
\hline Mujeres** & $173(145-202)$ & $163(134-178)$ & 0,0644 \\
\hline Hombres** & $153(138-191)$ & $143(132-185)$ & 0,0372 \\
\hline Colesterol HDL (mg/dL) ** & $40(35-44)$ & $38(33-48)$ & 0,6031 \\
\hline Mujeres & $41(36-45)$ & $41(35-48)$ & 0,5412 \\
\hline Hombres & $39,5(30,5-44)$ & $37(32-42)$ & 0,9088 \\
\hline Colesterol LDL (mg/dL) ** & $84(71-111,5)$ & $79(58-105)$ & 0,2546 \\
\hline Mujeres** & $89(76-121)$ & 79 (56-109) & 0,0961 \\
\hline Hombres** & 77 (59-99) & $80(61-104)$ & 0,8432 \\
\hline Triglicéridos $(\mathrm{mg} / \mathrm{dL}) * *$ & $179(117-248)$ & $141(118-206)$ & 0,0247 \\
\hline Mujeres** & $179(121-247)$ & $149(124-185)$ & 0,2273 \\
\hline Hombres** & $171,5(114-268)$ & $139(113,5-219,5)$ & 0,0546 \\
\hline
\end{tabular}

Valores expresados como valor absoluto (porcentaje). * Reportado como media (desviación estándar). ${ }^{* *}$ Reportado como mediana (rango intercuartilico). HbA1c\%: hemoglobina glicosilada. IMC: índice de masa corporal. n: población. M: mujeres. H: hombres. P<0,05.

y exenatide de corta acción, por el bajo número de pacientes con estas moléculas.

En relación al control metabólico, los resultados con análogos de GLP-1 han sido divergentes, con una tendencia clara en mejoría del perfil lipídico, descenso en c-LDL de hasta 15,4 $\mathrm{mg} / \mathrm{dL}$ y triglicéridos $(20,3 \mathrm{mg} / \mathrm{dL})^{(9,20,23)}$. Nosotros encontramos un cambio significativo en estas variables, con reducción de colesterol total y triglicéridos de 13,5 mg/dL y $38 \mathrm{mg} / \mathrm{dL}$, respectivamente, esta última ligeramente superior a la reportada en la literatura. En mujeres, se observó disminución en el nivel de c-LDL hasta de $10 \mathrm{mg} / \mathrm{dL}$, hallazgo no registrado en los hombres, en quienes tampoco se documentaron cambios significativos en c-HDL, dato resaltable, dado que al género femenino se le atribuye mayor dificultad en reducción de colesterol cuando se usa terapia estándar para el manejo de lí$\operatorname{pidos}^{(24)}$. 
Tabla 3. Manejo médico previo al inicio de análogos GLP-1, valores clínicos y paraclínicos posteriores al inicio de análogos GLP-1

\begin{tabular}{|c|c|c|c|c|c|c|c|c|c|c|}
\hline $\begin{array}{c}\text { Tratamiento } \\
\text { basal }\end{array}$ & $\begin{array}{l}N \\
69\end{array}$ & $\begin{array}{r}\text { HbA1 } \\
\text { Pre-GLP-1 }\end{array}$ & $\begin{array}{l}(\%)^{* *} \\
\text { ost GLP-1 }\end{array}$ & $\mathbf{p}$ & $\begin{array}{r}\text { Peso } \\
\text { Pre-GLP-1 }\end{array}$ & $\begin{array}{l}\text { g)** } \\
\text { st-GLP-1 }\end{array}$ & $\mathbf{p}$ & $\begin{array}{r}\text { Glucemia de } \\
\text { Pre-GLP-1 }\end{array}$ & $\begin{array}{l}\text { no }(\mathrm{mg} / \mathrm{dL} \text { )** } \\
\text { st-GLP-1 }\end{array}$ & $\mathbf{p}$ \\
\hline $\begin{array}{l}\text { Insulina basal } \\
+ \text { Bolos }\end{array}$ & 45 & $9,5(8,1-10,4)$ & $7,2(6,65-8,25)$ & 0 & $84(72-97)$ & $82(72-95)$ & 0 & $175,5(117-211)$ & $113(103-154)$ & 0,003 \\
\hline Insulina basal & 5 & $7,8(7,7-8,4)$ & $(6,8-7,1)$ &, 04 & $87(73-97)$ & 83,4 (71-95) & 0,04 & $152(1$ & 120-163) & 0,3 \\
\hline $\begin{array}{l}\text { No } \\
\text { tratamiento } \\
\text { farmacológico }\end{array}$ & 4 & $8,35(7,2-9,1)$ & $5,3(5,15-6,2)$ & 0,06 & $97(79,5-102)$ & $87(68-100)$ & 0,06 & $151(118-239)$ & $84(81,5-86,5)$ & 0,06 \\
\hline ADO & 15 & $7,8(6,9-8,5)$ & $6,8(6,4-7,9)$ & 0,04 & $83(73-87)$ & $82(68-88)$ & 0,1 & $135,5(102-153)$ & $114,5(104-136)$ & 0,008 \\
\hline
\end{tabular}

Valores expresados como valor absoluto (porcentaje). **Reportado como mediana (rango intercuartílico). DM2: diabetes mellitus tipo 2. N: Número de pacientes con tratamiento para DM2 al inicio de la formulación del análogo de GLP-1. ADO: antidiabético oral. HbA1c\%: hemoglobina glicosilada. P< 0,05.

Tabla 4. Datos clínicos y paraclínicos según análogo GLP-1

\begin{tabular}{|c|c|c|c|c|}
\hline Variable & $\begin{array}{l}N \\
65\end{array}$ & Pre- GLP-1 & Post- GLP-1 & $\mathbf{P}$ \\
\hline \multicolumn{5}{|l|}{ Peso $(\mathrm{Kg})^{* *}$} \\
\hline Liraglutide & 25 & $84(72-96)$ & 79 (71-93) & 0,0003 \\
\hline Exenatide XQ & 40 & $85(73-97,5)$ & $82(71,25-95)$ & 0,0007 \\
\hline \multicolumn{5}{|l|}{$\mathrm{HbA} 1 \mathrm{c} \%$ ** } \\
\hline Liraglutide & 25 & $8,4(6,9-9,7)$ & $7,1(6,6-8,1)$ & 0,00 \\
\hline Exenatide XQ & 40 & $9(7,9-9,9)$ & $7(6,65-7,7)$ & 0,00 \\
\hline \multicolumn{5}{|c|}{ Glucemia en ayunas $(\mathrm{mg} / \mathrm{dL})^{* *}$} \\
\hline Liraglutide & 25 & 155 (124-182) & $114(97-146)$ & 0,0003 \\
\hline Exenatide XQ & 40 & $141,5(112-200)$ & $112(98-143,5)$ & 0,0037 \\
\hline
\end{tabular}

Valores expresados como valor absoluto (porcentaje). **Reportado como mediana (rango intercuartílico). Análogos GLP-1: análogos del péptido similar al glucagón tipo 1. HbA1c\%: hemoglobina glicosilada. $\mathrm{P}<0,05$.

En investigaciones previas se ha observado que el manejo con análogos GLP-1 puede llevar a un descenso de la tensión arterial sistólica, $-0,5$ a $-3 \mathrm{mmHg}$, lo que para algunos autores resulta ser estadísticamente significativo ${ }^{(23)}$, en nuestro estudio no se encontraron cambios significativos en nivel de tensión arterial.

Los principales efectos adversos descritos con análogos de GLP-1 han sido náuseas, vómitos, diarrea y reacciones locales en el sitio de aplicación del medicamento ${ }^{(6,7,9)}$; en nuestro estudio se encontraron reacciones en $7 \%$ de los pacientes, en todos los casos náuseas, causa por la cual este grupo de pacientes debió abandonar el tratamiento a los tres meses de seguimiento.

El estudio tiene limitaciones, pues al ser retrospectivo con recopilación de datos basados en historias clínicas, se carece de información importante para el análisis, no encontramos información para determinar la indicación para el uso de uno u otro análogo de GLP-1 más que el juicio médico. Como tampoco fue analizada la adición de otras medicaciones, dieta y ejercicio que pudieran interferir con el desenlace.

\section{Conclusión}

El uso de análogos GLP-1 brinda una opción terapéutica en el manejo de pacientes con DM2, con reducción no solo de HbA1c, sino también en el peso y mejoría en el perfil lipídico condición encontrada en nuestro estudio y que coincide con los estudios a nivel mundial. Se deben realizar estudios para evaluar el mantenimiento de estos cambios a largo plazo. Los cambios observados en el perfil lipídico abren la puerta para hacer estudios con análisis de subgrupos al respecto.

\section{Agradecimientos}

Agradecemos a la universidad ICESI, a la Facultad de Medicina, postgrado de medicina interna y grupo de epidemiología e investigación, así como a la Fundación Clínica Valle del Lili, en Cali, Colombia, por permitir la ejecución de este trabajo. Un agradecimiento especial para Andrés Mauricio Castro, estadístico, y Robinson Pacheco, epidemiólogo del centro de investigación de la Fundación Valle del Lili. 


\section{Referencias}

1. Tamayo, DC. Diabetes en Colombia, descripción de la epidemiología actual. Observatorio de diabetes de Colombia, 2013;1-11.

2. UK Prospective Diabetes Study (UKPDS) Group. (1998). Intensive bloodglucose control with sulphonylureas or insulin compared with conventional treatment and risk of camplications in patients with type 2 diabetes (UKPDS 33). The Lancet, 1998; 352: 837-853.

3. ADVANCE Collaborative Group (2008). Intensive Blood Glucose Control and Vascular Outcomes in Patients with Type 2 Diabetes. N Engl J Med. 2008; (358), 2560- 2572.

4. William Duckworth Carlos Abraira, Thomas Moritz, Domenic Reda, Nicholas Emanuele, Peter D. Reaven, et al. Glucose Control and Vascular Complications in Veterans with Type 2 Diabetes. N Engl J Med. 2009; 360:129-39.

5. Buse, J, Drucker, D, Taylor K, Kim T, Walsh B, Hu H, et al. DURATION-1: exenatide once weekly produces sustained glycemic control and weight loss over 52 weeks. Diabetes Care. 2010; 33: 1255-1261.

6. Buse J, Nauck M, Forst T, SheZ W, Shenouda S, Heilmann C. et al. Exenatide once weekly versus liraglutide once daily in patients with type 2 diabetes (DURATION-6). A randomized open-label study. Lancet, 2013; 381: 117124.

7. Steven P. Marso, Gilbert H. Daniels, Kirstine Brown-Frandsen, Peter Kristensen, Johannes FE. Mann, Michael A. Nauck, y cols. For the LEADER Steering committee on behalf of the LEADER Trial Investigators. Liraglutide and Cardiovascular Outcomes in Type 2 Diabetes. N Engl J Med. 2016; 375:31122.

8. Pfeffer MA, Claggett B, Diaz R, Dickstein K, Gerstein HC, Køber LV, et al. for the ELIXA Investigators. Lixisenatide in Patients with Type 2 Diabetes and Acute Coronary Syndrome. N Engl J Med, 2015; 373:2247-57.

9. Buse J, Rosenstock J, Sesti G, Schmidt W, Montanya E, Brett J. et al. Liraglutide once a day versus exenatide twice a day for type 2 diabetes: a 26-week randomised, parallel-group, multinational, open-label trial (LEAD-6). Lancet, 2009; 374: 39-47.

10. American Diabetes Association. (2016). Standards of medical care in diabetes- 2016. Diabetes care, 39 (Suppl 1), S13-S22.

11. Zimmet P, Alberti KG, Shaw J. Global and societal implications of the diabetes epidemic. Nature. 2001; 414:782-787

12. Kumar PR, Bhansali A, Ravikiran M, et al. Utility of glycated hemoglobin in diagnosing type 2 diabetes mellitus: A community-based study. J Clin Endocrinol Metab 2010; 95:2832-2835

13. Análisis de Situación de Salud, COLOMBIA 2014. MINSALUD, Bogotá, D. C., Colombia, 2014, pp: 146-148
14. Sten Madsbad. Review of head-to-head comparisons of glucagon-like peptide-1 receptor agonists. Diabetes, Obesity and Metabolism 18: 317-332, 2016.

15. Stephen C L Gough, Bruce Bode, Vincent Woo, Helena W Rodbard, Sultan Linjawi, Pernille Poulsen et al. Efficacy and safety of a fixed-ratio combination of insulin degludec and liraglutide (IDegLira) compared with its components given alone: results of a phase 3, open-label, randomised, 26-week, treat-to-target trial in insulin-naive patients with type 2 diabetes. Lancet Diabetes Endocrinol 2014 Nov;2(11):885-93

16. C. Mathieu, H. W. Rodbard, B. Cariou, Y. Handelsman, A. Philis-Tsimikas, A M. Ocampo Francisco, et al. A comparison of adding liraglutide versus a single daily dose of insulin aspart to insulin degludec in subjects with type 2 diabetes (BEGIN: VICTOZA ADD-ON. Diabetes, Obesity and Metabolism 16: 636-644, 2014.

17. Marcus Lind, Irl B Hirsch, Jaakko Tuomilehto, Sofia Dahlqvist, Bo Ahrén, Ole Torffvit, et al. Liraglutide in people treated for type 2 diabetes with multiple daily insulin injections: randomised clinical trial (MDI Liraglutide trial) BMJ 2015;351: h5364

18. García de Lucas M, Olalla I, Sempere M. Liraglutida reduce biomarcadores y riesgo vascular en pacientes con diabetes mellitus tipo 2. Rev Med Chil 141(12): 1602-1604, dic. 2013.

19. Davidson JA, Orsted DD, Campos C. Efficacy and safety of liraglutide, a oncedaily human glucagon-like peptide- 1 analogue, in Latino/Hispanic patients with type 2 diabetes: post hoc analysis of data from four phase III trials. Diabetes, Obesity and Metabolism 18: 725-728, 2016.

20. Buse, J, Drucker, D, Taylor K, Kim T, Walsh B, Hu H, et al. DURATION-1: exenatide once weekly produces sustained glycemic control and weight loss over 52 weeks. Diabetes Care. 2010; 33: 1255-1261.

21. Du Q1, Wang YJ, Yang S, Zhao YY, Han P. Liraglutide for the Treatment of Type 2 Diabetes Mellitus: A Meta-analysis of Randomized Placebo-Controlled Trials. Adv Ther.2014. 31:1182-1195

22. Zachary t. Bloomgarden, Regina Dodis, Catherine m. Viscoli, Eric s. Holmboe, Silvio e. Inzucchi. Lower Baseline Glycemia Reduces Apparent Oral Agent Glucose-Lowering Efficacy. Diabetes care, volume 29, number 9, september 2006

23. Yu Mi Kang, Chang Hee Jung. Cardiovascular Effects of Glucagon-Like Peptide-1 Receptor Agonists. Endocrinol Metab 2016; 31:258-274

24. Russo G, Pintaudi B, Giorda C, Lucisano G, Nicolucci A, Cristofaro MR, et al. Age- and Gender-Related Differences in LDL-Cholesterol Management in Outpatients with Type 2 Diabetes Mellitus. Int I Endocrinol 2015;2015:957105 